\title{
NONLOCALITY OF ACCELERATED SYSTEMS
}

\author{
BAHRAM MASHHOON \\ Department of Physics and Astronomy, University of Missouri-Columbia, \\ Columbia, Missouri 65211, USA \\ E-mail: mashhoonb@missouri.edu
}

Dedicated to Prof. Remo Ruffini in honor of his 60th birthday

\begin{abstract}
The conceptual basis for the nonlocality of accelerated systems is presented. The nonlocal theory of accelerated observers and its consequences are briefly described. Nonlocal field equations are developed for the case of the electrodynamics of linearly accelerated systems.
\end{abstract}

\section{Introduction}

Einstein's general relativity provides a satisfactory description of gravitation at the classical level [1]; however, a theory of gravitation valid at the quantum level has been elusive. One way out of this situation would be to return to the physical foundations of general relativity and identify, from a physical standpoint, the main obstacle to quantization. The result of such an analysis [2-4] is that the hypothesis of locality is the first major stumbling block. This hypothesis provides the theoretical connection between accelerated and inertial observers in the special theory of relativity.

The fundamental quantum laws of physics have been formulated with respect to ideal inertial observers, while actual observers are all noninertial. It follows from the success of the quantum theory that there must be a direct connection between accelerated and inertial observers. What is the nature of this connection? The standard theory of relativity postulates a pointwise connection between actual and inertial observers. It states that an accelerated observer is equivalent, at each instant along its worldline, to an otherwise identical momentarily comoving inertial observer. This hypothesis of locality amounts to replacing the worldline by its tangent vector at each instant. The idea originates from Newtonian mechanics of point particles, where the accelerated particle and the momentarily comoving inertial particle have the same state. If all physical phenomena could be described in terms of pointlike coincidences, then the hypothesis of locality would be exactly valid.

Lorentz invariance together with the hypothesis of locality constitutes the physical basis of the special theory of relativity. Moreover, the hypothesis of locality provides the crucial link between the special and general theories of relativity : Einstein's heuristic principle of equivalence relates the measurements of an observer in a gravitational field with those of an accelerated observer in Minkowski spacetime; hence, this principle will lose its operational significance if one does not know what accelerated observers measure.

This work is primarily concerned with the nonlocal measurements of noninertial observers in Minkowski spacetime. Therefore, imagine such an observer following a worldline $x^{\mu}(\tau)$ in a global inertial frame with coordinates $x^{\mu}=(c t, x, y, z)$.

nonlocal: submitted to World Scientific on January 2, 2019 
Here $\tau$ is the proper time along the worldline such that $-d \tau^{2}=\eta_{\mu \nu} d x^{\mu} d x^{\nu}$, where $\eta_{\mu \nu}$ is the Minkowski metric tensor with signature +2 . The hypothesis of locality implies that the accelerated observer carries an orthonormal tetrad frame $\lambda^{\mu}{ }_{(\alpha)}$ such that $\lambda_{(0)}^{\mu}=d x^{\mu} / d \tau$ is the temporal axis and $\lambda^{\mu}{ }_{(i)}, i=1,2,3$, are the spatial axes of the local frame of the observer. The translational acceleration is defined via $A^{\mu}=d \lambda^{\mu}{ }_{(0)} / d \tau$, while the acceleration of the observer is given by the antisymmetric tensor $\Phi_{\alpha \beta}$,

$$
\frac{d \lambda^{\mu}(\alpha)}{d \tau}=\Phi_{\alpha}{ }^{\beta} \lambda_{(\beta)}^{\mu} .
$$

The "electric" part of the acceleration tensor $\Phi_{0 i}=a_{i}$ characterizes the translational acceleration vector, i.e. $a_{i}=A_{\mu} \lambda_{(i)}^{\mu}$, while the "magnetic" part of the acceleration tensor $\Phi_{i j}=\epsilon_{i j k} \Omega^{k}$ characterizes the rotational frequency $\boldsymbol{\Omega}$ of the local frame with respect to a local nonrotating (i.e. Fermi-Walker transported) frame. The acceleration of the observer is thus completely characterized by the spacetime scalars $\mathbf{a}(\tau)$ and $\boldsymbol{\Omega}(\tau)$, which determine the local rate of change of the state of the observer. To indicate the scale of such variation, it is useful to define acceleration lengths $\mathcal{L}=c^{2} / a$ and $c / \Omega$ as well as the corresponding acceleration times given by $\mathcal{L} / c$. If $\lambda$ is the intrinsic length scale of the phenomenon under observation, the deviation from the hypothesis of locality is expected to be proportional to $\lambda / \mathcal{L}$. If this deviation is so small as to be below the detection capability of the observer, then the hypothesis of locality is valid. This is indeed the case for most Earth-bound experiments, since $c^{2} / g_{\oplus} \simeq 1 \mathrm{lyr}$ and $c / \Omega_{\oplus} \simeq 28 \mathrm{AU}$. As a matter of principle, however, classical wave phenomena violate the hypothesis of locality. Consider, for instance, the reception of an electromagnetic wave by the accelerated observer; to determine the properties of the incident wave, the reception of a few oscillations are in any case necessary. The hypothesis of locality will hold if these oscillations have vanishing durations, i.e. in the ray limit $\lambda / \mathcal{L} \rightarrow 0$.

There is an analog of the correspondence principle at work here: The hypothesis of locality is necessary for the establishment of the reference frame of the observer as well as for the description of standard measuring devices. That is, inertial effects are always at work in any accelerated measuring device. If these inertial effects integrate to a measurable influence over the duration of elementary local observations, then the device may not be considered locally inertial. To detect nonlocal effects, however, one needs access to devices that are locally inertial.

Standard measuring devices are those that are consistent with the hypothesis of locality. The basic spacetime measurements of inertial observers involve ideal clocks and measuring rods; therefore, the corresponding measurements of accelerated observers involve standard clocks and standard measuring rods. Thus standard clocks measure proper time and standard infinitesimal measuring rods may be used to establish extended coordinate systems in space. The spatial determinations of ideal inertial observers are in accordance with Euclidean geometry; therefore, in Minkowski spacetime an accelerated observer at each instant along its worldline experiences the Euclidean space in accordance with the hypothesis of locality. On this basis, a geodesic coordinate system may be established along the worldline;

nonlocal: submitted to World Scientific on January 2, 2019 
however, this system naturally has spatial limitations due to the requirement of the admissibility of the geodesic coordinates. Moreover, the conceptual consistency of spatial determinations by accelerated observers has been critically examined by means of thought experiments with the conclusion that finite standard measuring rods do not, in principle, exist. More precisely, it is not possible to put infinitesimal standard measuring rods one next to another and so on in order to come up with a definite length for the distance between two points in space. In practice, only distances that are negligibly small compared to the relevant acceleration lengths may be unambiguously defined [2-5]. In view of these limitations, accelerated reference frames are eschewed in the nonlocal theory of accelerated observers. Furthermore, severe restrictions must be placed on any spatial dimension $D$ of a standard device; that is, $D<<\mathcal{L}$, where $\mathcal{L}$ is the relevant acceleration scale. These considerations are consistent with the pointwise nature of the hypothesis of locality.

The nonlocal theory of accelerated observers is described in section 2. Nonlocal electrodynamics of linearly accelerated systems is considered in section 3 and the nonlocal field equations for such observers are discussed in section 4 . Section 5 contains a brief discussion. For the sake of simplicity, units are chosen such that $c=1$ in the rest of this paper.

\section{Nonlocal theory of accelerated observers}

An accelerated observer along its worldline passes through a continuous infinity of hypothetical momentarily comoving inertial observers. The events along the worldline can be uniquely identified by the proper time $\tau$. Let $\psi(x)$ be a radiation field in the background Minkowski spacetime and $\hat{\psi}(\tau)$ be the field as measured by the hypothetical inertial observer along the worldline at $\tau$. At the same spacetime event, $\hat{\psi}=\Lambda \psi$, where $\Lambda$ belongs to a matrix representation of the Lorentz group. More precisely, suppose that $x^{\prime}=L x+s$ is a passive proper Poincaré transformation of the inertial spacetime coordinates; then, $\psi^{\prime}\left(x^{\prime}\right)=\Lambda(L) \psi(x)$, so that $\Lambda=1$ for a scalar field. Along the worldline, events are characterized by the proper time $\tau$; hence, $\hat{\psi}(\tau)=\Lambda(\tau) \psi(\tau)$ is the measured field in accordance with the hypothesis of locality.

The most general linear relationship between $\Psi(\tau)$, which is the field measured by the accelerated observer, and $\hat{\psi}(\tau)$ that is consistent with the requirement of causality is

$$
\Psi(\tau)=\hat{\psi}(\tau)+\int_{\tau_{0}}^{\tau} K\left(\tau, \tau^{\prime}\right) \hat{\psi}\left(\tau^{\prime}\right) d \tau^{\prime}
$$

where $\tau_{0}$ is the instant at which the acceleration begins. To avoid unphysical situations, the accelerated motion is generally assumed to have a finite duration. Here $K\left(\tau, \tau^{\prime}\right)$ is a kernel that is directly related to the acceleration of the observer, since in the absence of acceleration the kernel $K$ and hence the nonlocal part of equation (2) must vanish. Moreover, the nonlocal part is expected to vanish for $\lambda / \mathcal{L} \rightarrow 0$ and equation (2) would then reduce to the expression of the hypothesis of locality for field measurement. Thus equation (2) is consistent with the superposition principle

nonlocal: submitted to World Scientific on January 2, 2019 
and involves a weighted average of the field over the past worldline of the accelerated observer. This is in general agreement with the notion that field measurement does not occur at a point but involves a certain spacetime average $[6,7]$.

Equation (2) may be written as

$$
\Psi=\Lambda \psi+\int_{\tau_{0}}^{\tau} K\left(\tau, \tau^{\prime}\right) \Lambda\left(\tau^{\prime}\right) \psi\left(\tau^{\prime}\right) d \tau^{\prime}
$$

which is the general expression relating the field measured by the static inertial observers $\psi$ to the field measured by accelerated observers $\Psi$. Equation (2) is a Volterra integral equation relating $\hat{\psi}$ to $\Psi$. According to Volterra's theorem, in the space of continuous functions the relation between $\Psi$ and $\hat{\psi}$ (and hence $\psi$ ) is unique [8]. This theorem has been extended to the Hilbert space of squareintegrable functions by Tricomi [9]. The Volterra-Tricomi uniqueness result can be used in the determination of the kernel $K$.

The kernel $K$ is determined on the basis of the postulate that a basic radiation field can never stand completely still with respect to any observer [10]. This is a generalization of the well-known fact that a basic radiation field can never stand completely still with respect to an inertial observer. That is, in the Doppler formula $\omega^{\prime}=\gamma \omega(1-\mathbf{v} \cdot \hat{\mathbf{k}})$, the only way that $\omega^{\prime}$ can vanish is if $\omega$ vanishes. This condition can be implemented in the general case involving noninertial observers as well. Indeed, this requirement means that if $\Psi(\tau)$ should turn out to be a constant, then $\psi(\tau)$ must have been a constant in the first place. The Volterra-Tricomi uniqueness theorem then implies that for any true radiation field $\psi$, the measured field $\Psi$ will never be a constant. Since $\Psi\left(\tau_{0}\right)=\hat{\psi}\left(\tau_{0}\right)=\Lambda\left(\tau_{0}\right) \psi\left(\tau_{0}\right)$, equation (3) satisfies the desired condition - that if $\Psi$ is constant, then $\psi$ must be constant - once

$$
\Lambda\left(\tau_{0}\right)=\Lambda(\tau)+\int_{\tau_{0}}^{\tau} K\left(\tau, \tau^{\prime}\right) \Lambda\left(\tau^{\prime}\right) d \tau^{\prime}
$$

holds. A detailed examination of the solutions of equation (4) and the comparison of the results with observational data on spin-rotation coupling lead to the conclusion that the only acceptable kernel is of the form [11]

$$
K\left(\tau, \tau^{\prime}\right)=k\left(\tau^{\prime}\right)=-\frac{d \Lambda\left(\tau^{\prime}\right)}{d \tau^{\prime}} \Lambda^{-1}\left(\tau^{\prime}\right) .
$$

The kernel (5) is directly proportional to the acceleration of the observer and is in fact a simple solution of equation (4) as can be verified by direct substitution.

The general nonlocal relation (3) with the kernel (5) is expected to hold in the quantum domain as well, ensuring the independence of the number of the quanta of the field from the motion of the observer.

An immediate consequence of this nonlocal theory of accelerated systems may be noted : the kernel vanishes for a constant $\Lambda$. It follows from this fact that the nonlocal theory forbids the existence of a pure scalar (or pseudoscalar) radiation field. Such a field must therefore be a composite. This circumstance is consistent 
with experimental results available at present. Further observational consequences of the nonlocal theory have been discussed in [12].

To illustrate the general theory described in this section, the rest of this paper is devoted to nonlocal electrodynamics [13-15]. In particular, it is the purpose of this paper to derive the corresponding nonlocal field equations that would reduce to Maxwell's equations in the local limit.

\section{Nonlocal electrodynamics}

Consider the measurement of the electromagnetic field by a linearly accelerated observer. That is, at $\tau=\tau_{0}$ the observer accelerates from rest with acceleration $g(\tau)>0$ along the $z$-axis. For $\tau \geq \tau_{0}$, the orthonormal tetrad of the observer is given by

$$
\begin{aligned}
& \lambda^{\mu}{ }_{(0)}=(C, 0,0, S), \\
& \lambda^{\mu}{ }_{(1)}=(0,1,0,0), \\
& \lambda^{\mu}{ }_{(2)}=(0,0,1,0), \\
& \lambda^{\mu}{ }_{(3)}=(S, 0,0, C),
\end{aligned}
$$

where $C=\cosh \theta, S=\sinh \theta$ and

$$
\theta=\int_{\tau_{0}}^{\tau} g\left(\tau^{\prime}\right) d \tau^{\prime}
$$

Let $F_{\mu \nu}$ be the Faraday tensor of an electromagnetic radiation field in Minkowski spacetime. At each instant of proper time $\tau$ along the worldline, the momentarily comoving inertial observer measures

$$
\hat{F}_{\alpha \beta}(\tau)=F_{\mu \nu} \lambda_{(\alpha)}^{\mu} \lambda_{(\beta)}^{\nu} .
$$

Using the natural decomposition $F_{\mu \nu} \rightarrow(\mathbf{E}, \mathbf{B})$, where $F_{0 i}=-E_{i}$ and $F_{i j}=\epsilon_{i j k} B^{k}$, one can write equation (11) as $\hat{F}=\Lambda F$, where $F$ is a column 6 -vector with $\mathbf{E}$ and $\mathbf{B}$ as components and $\Lambda$ is a $6 \times 6$ matrix. Imagine the measurement of electric and magnetic fields via measuring devices carried by the accelerated observer. If the acceleration of the observer could be ignored over the length and time scales of the measurement, then — as shown by Bohr and Rosenfeld [6,7] for the case of ideal inertial observers - charged particle dynamics in accordance with the Lorentz force law would lead to the conclusion that one would effectively measure the average field given in equation (11) with a level of accuracy that would depend on the sensitivity of the measuring devices. On the other hand, the charged particles have been subject to the effects of acceleration since $\tau_{0}$, as the observer and its comoving devices are noninertial. The nonlocality of field measurement therefore implies that equation (11) should be considered a first approximation. To separate the essential aspect of this nonlocality from the limitations of measuring devices one needs the most general nonlocal relationship between the actually measured

nonlocal: submitted to World Scientific on January 2, 2019 
field and the field measured by the hypothetical momentarily comoving inertial observers. In this treatment, such a relationship is considered subject to the superposition principle, causality and the postulate that a basic radiation field would never stand completely still with respect to an observer. Thus the main equation of nonlocal electrodynamics is

$$
\hat{\mathcal{F}}(\tau)=\hat{F}(\tau)+\int_{\tau_{0}}^{\tau} k\left(\tau^{\prime}\right) \hat{F}\left(\tau^{\prime}\right) d \tau^{\prime}
$$

where $\hat{\mathcal{F}}$ is the field measured by the accelerated observer. It is possible to write this equation in the form

$$
\hat{F}(\tau)=\hat{\mathcal{F}}(\tau)+\int_{\tau_{0}}^{\tau} R\left(\tau, \tau^{\prime}\right) \hat{\mathcal{F}}\left(\tau^{\prime}\right) d \tau^{\prime}
$$

where $R\left(\tau, \tau^{\prime}\right)$ is the resolvent kernel associated with $k\left(\tau^{\prime}\right)$.

In the case under consideration, one can show that

$$
\Lambda=\left[\begin{array}{cc}
U & V \\
-V & U
\end{array}\right], \quad U=\left[\begin{array}{lll}
C & 0 & 0 \\
0 & C & 0 \\
0 & 0 & 1
\end{array}\right], V=S I_{3},
$$

where $I_{i},\left(I_{i}\right)_{j k}=-\epsilon_{i j k}$, is a $3 \times 3$ matrix that is proportional to the operator of infinitesimal rotations about the $x^{i}$-axis. Here $U V=V U=C S I_{3}$ and $U^{2}+V^{2}=I$, where $I$ is the unit matrix. Using equation (5), the kernel $k$ is given by

$$
k=-g(\tau)\left[\begin{array}{cc}
0 & I_{3} \\
-I_{3} & 0
\end{array}\right] .
$$

For the sake of simplicity, it will henceforth be assumed that $g(\tau)$ is constant, $\tau_{0}=0$ and thus $\theta=g \tau$. For a constant kernel $k$, the resolvent kernel $R\left(\tau, \tau^{\prime}\right)$ turns out to be of the convolution (Faltung) type, $R\left(\tau, \tau^{\prime}\right)=r\left(\tau-\tau^{\prime}\right)$, where $r$ may be expressed as $r(u)=-k \exp (-u k)$. In general, $R\left(\tau, \tau^{\prime}\right)$ could be very complicated; indeed, the manageable expression for $r$ is due to the assumption of uniform acceleration. Therefore in the simple case under consideration here the resolvent kernel turns out to be

$$
r(\tau)=g\left[\begin{array}{cc}
\mathrm{SJ}_{3} & \mathrm{CI} I_{3} \\
-\mathrm{CI}_{3} & \mathrm{SJ}_{3}
\end{array}\right],
$$

where $\left(J_{k}\right)_{i j}=\delta_{i j}-\delta_{i k} \delta_{j k} \quad, \quad J_{3}=-I_{3}{ }^{2}$ and $J_{3} I_{3}=I_{3} J_{3}=I_{3}$. It follows that equation (13) may be used to express the Faraday tensor $F_{\mu \nu}$ in terms of the nonlocal electromagnetic field of a class of accelerated observers. Since $F_{\mu \nu}$ satisfies Maxwell's equations, one can use equation (13) to derive the corresponding nonlocal field equations.

nonlocal: submitted to World Scientific on January 2, 2019 


\section{Nonlocal Maxwell's equations}

Consider the class of fundamental inertial observers in the background global system: Each observer is at rest at an event $x^{\mu}=(t, \mathbf{x})$ and carries an orthonormal frame whose axes coincide with those of the background inertial system. At $t=0$ the whole class is accelerated from rest along the $z$-axis with uniform acceleration $g$. Thus at any time $t>0$, an event $(t, x, y, z)$ is occupied by an accelerated observer in hyperbolic motion that at $t=0$ occupied $\left(0, x, y, z_{0}\right)$ such that

$$
z_{0}=z+\frac{1}{g}-\sqrt{t^{2}+\frac{1}{g^{2}}} .
$$

At each such event $(t, x, y, z)$, one can define a field $\mathcal{F}(t, x, y, z)$ such that $\hat{\mathcal{F}}(\tau)=$ $\Lambda \mathcal{F}$, where $\tau$ is the proper time, $g t=\sinh g \tau$, along the hyperbolic worldline. This would correspond to defining a spacetime tensor $\mathcal{F}_{\mu \nu} \rightarrow(\mathcal{E}, \mathcal{B})$ such that

$$
\hat{\mathcal{F}}_{\alpha \beta}=\mathcal{F}_{\mu \nu} \lambda_{(\alpha)}^{\mu} \lambda_{(\beta)}^{\nu} .
$$

Equation (13) may now be written in the following general form

$$
F(\tau)=\mathcal{F}(\tau)+\int_{\tau_{0}}^{\tau}\left[\Lambda^{-1}(\tau) R\left(\tau, \tau^{\prime}\right) \Lambda\left(\tau^{\prime}\right)\right] \mathcal{F}\left(\tau^{\prime}\right) d \tau^{\prime}
$$

In the case under consideration one finds that

$$
\Lambda^{-1}(\tau) r\left(\tau-\tau^{\prime}\right) \Lambda\left(\tau^{\prime}\right)=-k
$$

where $\Lambda(u)=\exp (-u k)$, which follows from equation (5) and the fact that $\Lambda(0)$ is the unit matrix. Thus equation (19) takes the form

$$
F(t, x, y, z)=\mathcal{F}(t, x, y, z)+g u_{0}(t)\left[\begin{array}{cc}
0 & I_{3} \\
-I_{3} & 0
\end{array}\right] \int_{0}^{\tau} \mathcal{F}\left(t^{\prime}, x, y, z^{\prime}\right) d \tau^{\prime},
$$

where the unit step function $u_{0}(t), u_{0}=1$ for $t>0$ and $u_{0}=0$ otherwise, has been introduced to ensure nonlocality only for $t>0$. Using $\sinh g \tau=g t$, one can write $g d \tau=d t / \zeta(t)$, where

$$
\zeta(t)=\sqrt{t^{2}+\frac{1}{g^{2}}} .
$$

Moreover, in equation (21), $z^{\prime}=z_{0}-g^{-1}+\zeta\left(t^{\prime}\right)$, where $z_{0}=z_{0}(t, z)$ is given by equation (17). It follows that

$$
F(t, \mathbf{x})=\mathcal{F}(t, \mathbf{x})+u_{0}(t)\left[\begin{array}{cc}
0 & I_{3} \\
-I_{3} & 0
\end{array}\right] \int_{0}^{t} \mathcal{F}\left(t^{\prime}, x, y, z-\zeta+\zeta^{\prime}\right) \frac{d t^{\prime}}{\zeta^{\prime}},
$$

where $\zeta$ and $\zeta^{\prime}$ stand for $\zeta(t)$ and $\zeta\left(t^{\prime}\right)$, respectively. This equation may be expressed as

nonlocal: submitted to World Scientific on January 2, 2019 


$$
\begin{aligned}
& \mathbf{E}=\mathcal{E}+u_{0}(t) \hat{\mathbf{z}} \times \int_{0}^{t} \mathcal{B}\left(t^{\prime}, x, y, z-\zeta+\zeta^{\prime}\right) \frac{d t^{\prime}}{\zeta^{\prime}}, \\
& \mathbf{B}=\mathcal{B}-u_{0}(t) \hat{\mathbf{z}} \times \int_{0}^{t} \mathcal{E}\left(t^{\prime}, x, y, z-\zeta+\zeta^{\prime}\right) \frac{d t^{\prime}}{\zeta^{\prime}},
\end{aligned}
$$

so that the fields are unchanged parallel to the direction of the motion of the observer.

Introducing the Kramers vectors $\mathbf{W}^{ \pm}=\mathbf{E} \pm i \mathbf{B}$, the source-free Maxwell equations can be written in the form

$$
\begin{gathered}
\boldsymbol{\nabla} \cdot \mathbf{W}^{ \pm}=0, \\
\frac{1}{i} \nabla \times \mathbf{W}^{ \pm}= \pm \frac{\partial}{\partial t} \mathbf{W}^{ \pm} .
\end{gathered}
$$

When dealing with complex fields, $\mathbf{W}^{+}$represents a positive-helicity wave and $\mathbf{W}^{-}$ represents a negative-helicity wave. Equations (24) and (25) can be expressed as

$$
\mathbf{W}^{ \pm}=\mathcal{W}^{ \pm} \mp i u_{0}(t) \hat{\mathbf{z}} \times \int_{0}^{t} \mathcal{W}^{ \pm}\left(t^{\prime}, x, y, z-\zeta+\zeta^{\prime}\right) \frac{d t^{\prime}}{\zeta^{\prime}}
$$

where $\mathcal{W}^{ \pm}=\mathcal{E} \pm i \mathcal{B}$. Thus the nonlocal field equations are given by

$$
\begin{aligned}
& \nabla \cdot \mathcal{W}^{ \pm}=\mp i u_{0}(t) \hat{\mathbf{z}} \cdot \int_{0}^{t} \nabla \times \mathcal{W}^{ \pm}\left(t^{\prime}, x, y, z-\zeta+\zeta^{\prime}\right) \frac{d t^{\prime}}{\zeta^{\prime}} \\
& \frac{1}{i}\left(\nabla-\frac{u_{0}(t)}{\zeta} \hat{\mathbf{z}}\right) \times \mathcal{W}^{ \pm}= \pm \frac{\partial}{\partial t} \mathcal{W}^{ \pm} \\
& \pm u_{0}(t) \int_{0}^{t} \nabla \times\left(\hat{\mathbf{z}} \times \mathcal{W}^{ \pm}\right)\left(t^{\prime}, x, y, z-\zeta+\zeta^{\prime}\right) \frac{d t^{\prime}}{\zeta^{\prime}} \\
&+i \frac{t u_{0}(t)}{\zeta} \hat{\mathbf{z}} \times \int_{0}^{t} \partial_{z} \mathcal{W}^{ \pm}\left(t^{\prime}, x, y, z-\zeta+\zeta^{\prime}\right) \frac{d t^{\prime}}{\zeta^{\prime}}
\end{aligned}
$$

Just as in equations (26) and (27), $\mathcal{W}^{+}$and $\mathcal{W}^{-}$satisfy separate equations, i.e. nonlocality in general treats $\mathcal{W}^{+}$and $\mathcal{W}^{-}$differently, but cannot convert one helicity state into another. Moreover, equations (26) and (27) can be combined in the standard manner to yield the wave equations $\square \mathbf{W}^{ \pm}=0$. A similar procedure in the case of equations (29) and (30)-or, alternatively, applying the d'Alembertian operator directly to equation (28) - results in rather complicated and unwieldy wave equations for $\mathcal{W}^{ \pm}$.

nonlocal: submitted to World Scientific on January 2, 2019 


\section{Discussion}

Nonlocal field equations (29) and (30) have been derived in the background global inertial frame. These equations are Lorentz invariant, since they originate from the manifestly Lorentz-invariant nonlocal ansatz (2). Moreover, it is possible to transform the nonlocal field equations to any other coordinate system using the standard approach based on the invariance of the 2 -form $\mathcal{F}_{\mu \nu} d x^{\mu} \wedge d x^{\nu}=\mathcal{F}_{\rho \sigma}^{\prime} d x^{\rho} \wedge$ $d x^{\prime \sigma}$.

The approach adopted in this paper for the development of nonlocal field equations is quite general. The resulting equations can be rather complicated, however. Thus attention has been confined to hyperbolic motion. An infinite amount of energy is required to sustain an observer in hyperbolic motion over an infinite time interval; nevertheless, the case of uniform linear acceleration has been treated ex-

plicitly in this work for the sake of simplicity.

\section{References}

1. A. Einstein, The Meaning of Relativity (Princeton University Press, Princeton, 1950).

2. B. Mashhoon, Phys. Lett. A 143 (1990) 176.

3. B. Mashhoon, Phys. Lett. A 145 (1990) 147.

4. B. Mashhoon, "Measurement Theory and General Relativity," in: Black Holes: Theory and Observation, edited by F. W. Hehl, C. Kiefer and R. Metzler (Springer, Berlin, 1998), pp. 269-284.

5. B. Mashhoon and U. Muench, Ann. Phys. (Leipzig) 11 (2002) 532.

6. N. Bohr and L. Rosenfeld, Det. Kgl. dansk. Vid. Selskab. 12 (1933) no. 8, translated in: Quantum Theory and Measurement, edited by J. A. Wheeler and W. H. Zurek (Princeton University Press, Princeton, 1983).

7. N. Bohr and L. Rosenfeld, Phys. Rev. 78 (1950) 794.

8. V. Volterra, Theory of Functionals and of Integral and Integro-Differential Equations (Dover, New York, 1959).

9. F. G. Tricomi, Integral Equations (Interscience, New York, 1957).

10. B. Mashhoon, Phys. Rev. A 47 (1993) 4498.

11. C. Chicone and B. Mashhoon, Phys. Lett. A 298 (2002) 229.

12. B. Mashhoon, Phys. Lett. A 306 (2002) 66.

13. B. Mashhoon, "Nonlocal Electrodynamics," in: Cosmology and Gravitation, edited by M. Novello (Editions Frontières, Gif-sur-Yvette, 1994), pp. 245-295.

14. U. Muench, F. W. Hehl and B. Mashhoon, Phys. Lett. A 271 (2000) 8.

15. F. W. Hehl and Y. N. Obukhov, Foundations of Classical Electrodynamics (Birkhäuser, Boston, 2003). 